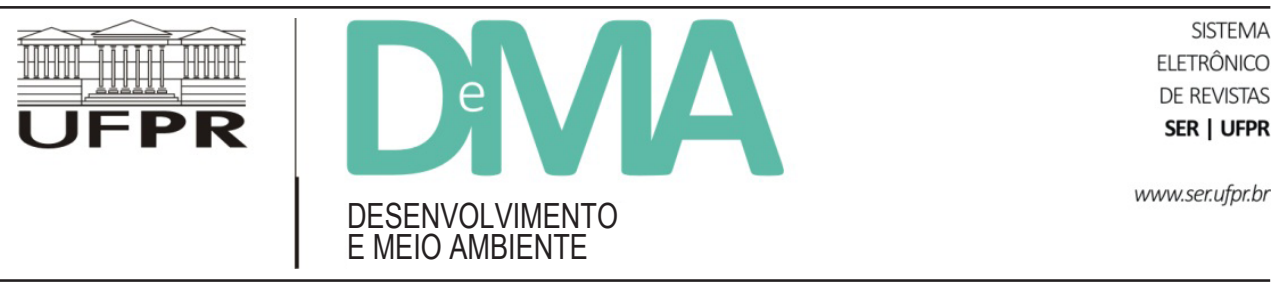

\title{
Farmacopeia natural de comunidades rurais no Estado do Piauí, Nordeste do Brasil
}

\section{Natural Pharmacopeia of Rural Communities in the State of Piauí, Northeast of Brazil}

\author{
Maria Pessoa da SILVA ${ }^{1 *}$, Roseli Farias Melo de BARROS ${ }^{1}$, José Machado MOITA NETO ${ }^{1}$ \\ ${ }^{1}$ Programa de Pós-Graduação em Desenvolvimento e Meio Ambiente (PRODEMA), Universidade Federal do Piauí (UFPI), Teresina, PI, Brasil. \\ *E-mail de contato: cruzinhabio@yahoo.com.br
}

Artigo recebido em 26 de julho de 2014, versão final aceita em 6 de abril de 2015.

RESUMO: Objetivou-se conhecer as plantas medicinais, as formas de usos e indicações terapêuticas atribuídas pelas comunidades Pau-Arrastado, Salinas e Resolvido. Utilizaram-se entrevista semiestruturada e turnês guiadas e seguiu-se metodologia botânica usual. Amostraram-se 93 pessoas (51,9\% do total). As espécies foram agrupadas em 18 categorias de doenças, de acordo com a Organização Mundial de Saúde (OMS). Calculou-se o Fator de Consenso dos Informantes (FCI) e a Importância Relativa (IR). Referiram-se 81 espécies, em 40 famílias botânicas e 69 gêneros, sendo $75,3 \%$ nativas. A família mais destacada foi Fabaceae $(18,5 \%)$, as folhas foram as partes mais usadas (32\%) e o preparo mais adotado foi a tintura $(38,2 \%)$. Destacaram-se os sistemas corporais: agentes de infecções bacterianas e virais $(27,2 \%)$, doenças do aparelho digestivo $(22,4 \%)$ e genitourinário (19,5\%). Croton campestris A. St. Hil. e Ximenia americana L. foram as espécies mais versáteis, com IR $(2,00)$. Houve maior consenso para as plantas indicadas no tratamento de doenças do fígado.

Palavras-chave: etnobotânica; Campo Maior; comunidades rurais.

ABSTRACT: The purpose of this work is to know more about medicinal plants, forms of usage and therapeutic indications determined by the communities of Pau-Arrastado, Salinas and Resolvido. We used semi-structured interviews, guided tours and followed the usual botanical methodology. The study sample included 93 individuals (51.9\% of the total). Species were grouped into 18 categories of disease, according to the World Health Organization (WHO). We calculated the Factor of Informant Consensus (FIC) and the Index of Relative Importance (IRI). They included eighty-one (81) species, belonging to 40 botanical families and 69 genera were referred, of which $75.3 \%$ were native ones. The most prominent family was Fabaceae $(18.5 \%)$, and the leaves were the most used parts (32\%). The preparation method most used was dye (38.2\%). The highlights were the body systems: agents of bacterial and viral infections (27.2\%), digestive system diseases (22.4\%) and genitourinary diseases (19.5\%). Croton campestris A. St. Hil. and Ximenia americana L. were the most versatile with an IRI of 2.00. There was a greater consensus for the plants indicated for the treatment of liver diseases.

Keywords: ethnobotany; Campo Maior; rural communities. 


\section{Introdução}

A importância dos sistemas terapêuticos tradicionais vem desde a antiguidade (Rocha et al., 2014). A sociedade humana acumula um acervo de informações sobre o ambiente que a cerca, que lhe possibilita interagir com ele para prover suas necessidades de sobrevivência. O conhecimento tradicional sobre o uso das plantas é vasto e, em muitos casos, o único recurso terapêutico disponível às populações rurais e grupos étnicos de países em desenvolvimento, tais como o Brasil (Maciel et al., 2002; Pasa et al., 2005; Agra et al., 2008).

Segundo a Política Nacional de Plantas Medicinais e Fitoterápicas, as plantas medicinais são consideradas estratégicas para o fortalecimento da agricultura familiar, geração de emprego e renda, uso sustentável da biodiversidade brasileira, avanço tecnológico e melhoria da atenção à saúde da população brasileira (Brasil, 2006). Haller-Jr. (1982) define farmacopeia como os registros de compostos e substâncias de origem vegetal, animal ou mineral com propriedades terapêuticas reconhecidas cientificamente, com suas respectivas preparações e formas de uso.

Amorozo (1996), Di Stasi (1996) e Guarim Neto \& Maciel (2008) relatam que os estudos com plantas medicinais têm sido responsáveis por inúmeras e importantes descobertas e afirmam que no Brasil $20 \%$ da população consome $63 \%$ dos medicamentos alopáticos disponíveis e o restante encontra-se nos produtos de origem natural, especialmente nas plantas medicinais, muitas vezes a única fonte de recurso terapêutico e uma rica herança cultural local (Giraldi \& Hanazaki, 2010).

Diversas pesquisas com plantas medicinais trouxeram uma contribuição significativa na área fitoterápica e farmacológica, onde podemos citar as de Pereira et al. (2007), que visaram conhecer as plantas nativas comercializadas por ervateiros no Cerrado da região de Dourados (MS), e Silva \& Proença (2008), que realizaram um levantamento etnobotânico das plantas medicinais usadas por comunidades rurais e urbanas no município de Ouro Verde (GO), visando eleger espécies nativas do bioma Cerrado com potenciais para estudos farmacológicos e fitoterápicos.
No Nordeste brasileiro nos últimos tempos, vários núcleos de pesquisas oriundos de Instituições de Ensino Superior, como a Universidade Federal Rural de Pernambuco (UFRPE), a Universidade Federal de Pernambuco (UFPE), a Universidade Federal da Paraíba (UFPB) e a Universidade Federal do Piauí (UFPI), têm desenvolvido pesquisas com plantas medicinais, tanto nos aspectos etnobotânico, etnofarmacológico, fitoquímico, farmacognóstico quanto no farmacológico. Alguns trabalhos desenvolvidos no Piaúi envolvendo plantas medicinais do Cerrado foram efetivados pelos seguintes autores: Aguiar \& Barros (2012), que objetivaram realizar um inventário das plantas medicinais e conhecer o emprego e a importância dessas espécies cultivadas em quintais do município de Demerval Lobão (PI), e Sousa et al. (2012), que pesquisaram o conhecimento botânico compartilhado entre homens e mulheres numa comunidade de pescadores da Área de Proteção Ambiental Delta do Parnaíba. As implicações dessa nova postura são refletidas no grande número de publicações com esses enfoques em periódicos nacionais e internacionais.

Neste sentido, o presente trabalho objetivou estudar a relação que os membros das comunidades rurais inseridas em um ambiente de transição, com predominância em Cerrado, têm com a vegetação de suas áreas de convivência e realizar o levantamento das plantas utilizadas como medicamentos, identificando sua utilização nos processos de produção diária de remédios naturais, bem como as indicações terapêuticas atribuídas pelos moradores das comunidades.

\section{Material e método}

\subsection{Descrição da área}

$\mathrm{O}$ estudo foi realizado em comunidades rurais do município de Campo Maior, PI (0449'40" S e 42 $10^{\circ}$ '07" W), distante da capital cerca de $84 \mathrm{~km}$ (Figura 1), com uma área de $1.699,383 \mathrm{~km}^{2}$ e uma população de 45.827 habitantes, correspondendo a uma densidade demográfica de 24,4 habitantes $/ \mathrm{km}^{2}$, onde $25,96 \%$ das pessoas habitam a zona rural. A maior altitude apresentada no centro da cidade atinge $125 \mathrm{~m}$ e as planícies predominam 

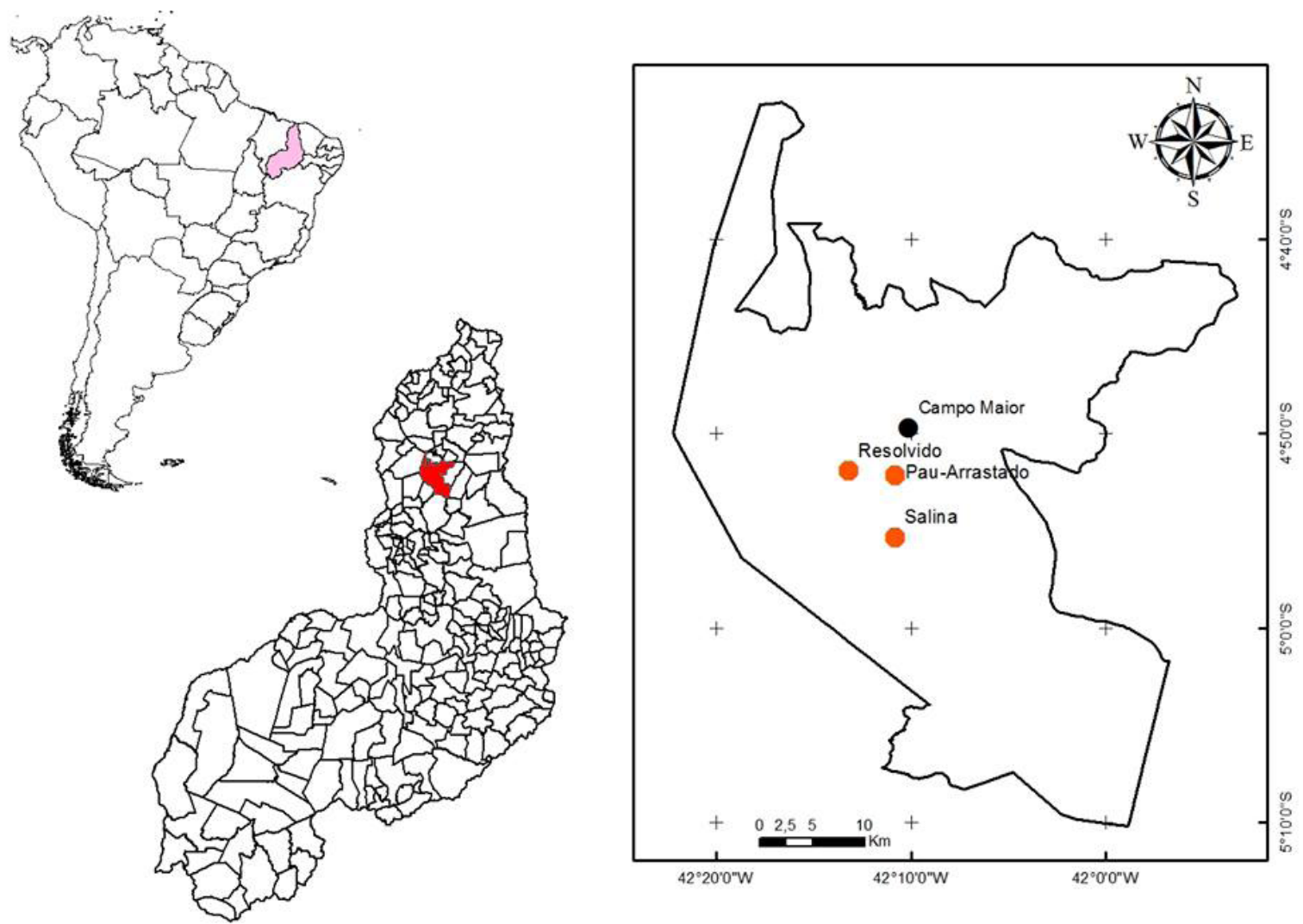

FIGURA 1 - Localização do município de Campo Maior/PI e das comunidades Pau-Arrastado, Salinas e Resolvido. FONTE: IBGE (2008), modificado por Michel Francisco da Cunha Coelho em 2015.

na bacia sedimentar do Meio Norte, sendo sua baixada no rio Longá a de maior significação, onde há zonas intercaladas de "cuestas", com chapadas de altitudes de 150 a 300 metros na parte leste, onde ocorre a Serra de Campo Maior, distante do centro aproximadamente 15 km (IBGE, 2008).

As chuvas concentram-se no período de janeiro a abril, com precipitação anual de $1.280,8 \mathrm{~mm}$, temperatura média de $33,7^{\circ} \mathrm{C}$, tendo a mínima de $23,4^{\circ} \mathrm{C}$ em janeiro e a máxima de $38,7^{\circ} \mathrm{C}$ em novembro. Os compartimentos geomorfológicos na área refletem uma ação conjunta e sinérgica de geologia, clima, vegetação e hidrografia na forma de planícies fluviais instaladas entre terrenos elevados e morros isolados em contraste com os contrafortes e reversos da Serra de Campo Maior, Serra do Bugarim e Serra do Passa-Tempo (Barros et al., 2007).
A flora é de transição cerrado/caatinga e cerrado/ mata, com vegetação caducifólia e subcaducifólia estacionais. A vegetação herbácea é predominante, com fisionomia lembrando a savana africana, ocorrendo também à presença de carnaubais em planície inundáveis: savanas de Copernicia. Nas partes mais altas, não inundáveis, há vegetação arbustivo-arbórea com elementos de cerrado (Velloso et al., 2002).

O estudo foi realizado em três comunidades rurais localizadas no entorno Serra de Campo Maior: Pau-Arrastado $\left(04^{\circ} 53^{\prime} 0,5^{\prime \prime} \mathrm{S}\right.$ e $\left.42^{\circ} 10^{\prime} 17,9^{\prime \prime} \mathrm{W}\right)$, Salinas $\left(04^{\circ} 59^{\prime} 59,9^{\prime}\right.$ ' S e $42^{\circ} 10^{\prime} 24,2^{\prime}$ "W) e Resolvido

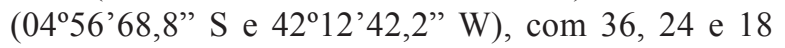
famílias, respectivamente (IBGE, 2008). As comunidades foram selecionadas como áreas de estudo pelas distinções geográficas, pela predominância da vegetação 
de transição e pelas diferentes atividades econômicas e de subsistência adotadas pelas mesmas.

\subsection{Coleta de dados}

O projeto de pesquisa desse estudo foi submetido ao Comitê de Ética e Pesquisa da Universidade Federal do Piauí (CAAE - 0088.0.045.000-09) e foi solicitada assinatura dos entrevistados no Termo de Consentimento Livre e Esclarecido (TCLE). As coletas botânicas e a herborização do material em estádio reprodutivo foram procedidas de acordo com a metodologia proposta por Mori et al. (1989). Todo o material encontra-se incorporado no acervo do Herbário Graziela Barroso (TEPB) da Universidade Federal do Piauí (UFPI). A identificação do material botânico foi procedida mediante análises morfológicas, consultas a bibliografias específicas, comparações com os materiais incorporados ao acervo do TEPB e confirmados por especialistas. Para a listagem dos táxons seguiu-se o APG III (2009). Consultou-se o site do MOBOT (2014), no intuito de corrigir a grafia dos nomes das espécies e abreviaturas dos autores botânicos.

A definição da amostra de pessoas entrevistadas foi estabelecida conforme a proposta de Begossi et al. (1993), que afirmam ser uma amostra representativa em comunidades com mais de 50 pessoas a proporção de $25 \%$ a $80 \%$. As comunidades Pau-Arrastado, Salinas e Resolvido, compostas por 36, 24 e 18 famílias, respectivamente (IBGE, 2008), contabilizaram um universo de 99, 78 e 76 pessoas residentes em cada comunidade, das quais 93 pessoas foram entrevistadas ( 54 mulheres e 39 homens), com idades que variaram de 18 a 83 anos, perfazendo $51,9 \%$ da população total acima de 18 anos, que corresponde a 179 pessoas. Definiu-se um número máximo de três pessoas entrevistadas por domicílio, sendo um jovem, um dos cônjuges ou ambos e um idoso, caso estivesse presente na composição familiar, visando verificar o conhecimento etnobotânico por faixa etária e gênero. A faixa etária foi definida de acordo com o IBGE (2009): jovens (18 a 24 anos); adultos ( 25 a 59 anos) e idosos (a partir de 60 anos).

Para obtenção dos dados socioeconômicos e etnobotânicos, foi adotada entrevista semiestruturada, com aplicação de formulário padronizado (Appolinário, 2006), contendo questões abertas e fechadas, que seguiram a ordem: identificação, localização, dados socioeconômicos (composição familiar, idade, gênero, escolaridade, renda, habitação, saneamento, religião) e a técnica turnê-guiada (Bernard, 1988), com o intuito de checar os nomes vulgares e realizar as coletas do material referenciado em estádio reprodutivo.

\subsection{Análise e tabulação dos dados}

$\mathrm{Na}$ análise quantitativa adotou-se o Índice de Importância Relativa (IR) e o Fator de Consenso dos Informantes (FCI). O IR foi calculado baseando-se na proposta de Bennett e Prance (2000), onde o valor máximo do IR obtido por uma espécie será 2 , através da fórmula $\mathrm{IR}=$ $\mathrm{NSC}+\mathrm{NP}$, onde NSC é igual ao número de sistemas corporais tratados por uma determinada espécie (NSCE), dividido pelo número total de sistemas corporais tratados pela espécie mais versátil (NSCEV); NP coincide com a relação entre o número de propriedades atribuídas a uma determinada espécie (NPE) e o número total de propriedades atribuídas à espécie mais versátil (NPEV). O FCI foi calculado com base na proposta de Trotter e Logan (1986), com a finalidade de identificar as categorias de doenças que apresentam maior importância relativa local, sendo obtido pela fórmula FCI $=n_{u r}-n_{t} / n_{u r}-1$, onde $n_{u r}$ é o número de citações de usos em cada categoria e $n_{t} \mathrm{o}$ número de espécies usadas.

\section{Resultados e discussão}

Foram citadas 81 espécies referidas como medicinais, em 342 citações, distribuídas em 40 famílias botânicas e 69 gêneros (Tabela 1). As famílias botânicas mais representativas em número de espécies, que apresentaram padrões condizentes com outros trabalhos realizados no Brasil, foram: Fabaceae (18,5\%), Lamiaceae $(11,1 \%)$ e Euphorbiaceae $(7,4 \%)$. Estas famílias tiveram maior número de citações nos resultados obtidos por Brito \& Brito (1993) e Silva \& Andrade (2005). Kruel \& Peixoto (2004) e Almeida et al. (2006) incluem a família Fabaceae entre as que contribuíram com o maior número de espécies medicinais. No Piauí, Franco \& Barros (2006) apresentaram o mesmo resultado para a família. Geralmente, nas pesquisas realizadas com plantas medicinais, a família Lamiaceae se destaca, por apresentar diversas espécies exóticas facilmente domesticadas, 
como apontado no trabalho realizado na Turquia por Fujita et al. (1995), onde foram referidas nove espécies para esta família.

As espécies utilizadas como medicinais nas comunidades estudadas crescem espontaneamente em ambientes naturais, antropicamente modificados ou em quintais, distribuindo-se nos estratos arbóreo (37,3\%), herbáceo (23\%), subarbustivo $(19,2 \%)$, arbustivo $(12,8 \%)$, liana $(5,1 \%)$ e arbustivo-escandente $(2,5 \%)$. Resultado semelhante quanto à porcentagem nos hábitos arbóreos e herbáceos foi referido por Rajakumar \& Shivanna (2009), que desenvolveram estudos sobre as plantas medicinais utilizadas na Índia. O uso das espécies arbóreas na área em estudo deve-se ao fato de estar inserida na zona rural, onde se observa uma dependência e uma interação maior por parte dos moradores com as mesmas nas atividades corriqueiras diárias, como: plantio, cozimento de alimentos, produção manufatureira, construção e produção de remédios naturais. Os moradores das três comunidades exploram todos os ambientes da região, demonstrando dinamismo e adaptação aos diversos habitats. O cercado e as matas são os ambientes mais explorados para coleta e cultivo das espécies medicinais, ficando o primeiro no domínio feminino e o segundo no dos homens.

As mulheres têm um papel fundamental no cultivo e uso das plantas e na manutenção dos quintais, pois são as principais mantenedoras destes e possuem um conhecimento mais específico de ervas e arbustos utilizados na terapia caseira, pois são as espécies cultivadas ou que nascem espontaneamente nas imediações das residências e cercados, enquanto que para os homens o conhecimento é maior das espécies arbóreas e arbustivas que geralmente encontram-se nos caminhos de roças, matas e no deslocamento até os locais de cultivo, fato também referido por Paulilo (2009), Schweickardt (2010) e Freitas et al. (2012), que confirmaram a importância feminina nos processos de desenvolvimento.

O tempo de moradia dos entrevistados nas comunidades é um fator extremamente relevante para a garantia das relações sociais, familiares e de interação com os bens naturais e nas três comunidades esse item atingiu uma média de 64,6 anos. Os moradores entrevistados da comunidade Pau-Arrastado chegaram a uma permanência mínima de dois anos e uma máxima de 83 anos, Salinas entre quatro e 63 anos e em Resolvido o tempo de moradia dos entrevistados foi entre um ano e 48 anos e meio.

Os entrevistados que forneceram mais informações sobre o uso medicinal de plantas estão distribuídos nas faixas etárias idosa $(66,6 \%)$ e adulta $(33,4 \%)$, fato que converge com a maioria dos dados apresentados por Abreu (2000), em Amarante-PI, Albuquerque \& Albuquerque (2005) em Vila Velha-PE, Almeida et al. (2006), no semiárido nordestino, e Franco \& Barros (2006), em Esperantina-PI. Pode-se justificar a faixa etária idosa e adulta pelo fato do tempo de convivênvia com o recurso e que, com o passar dos anos, os moradores vão adquirindo mais experiência e também respeito nas comunidades, sendo assim indicados com notório saber e reconhecidos pelos demais moradores.

As espécies medicinais nativas utilizadas nas comunidades corresponderam a um percentual de 75,3\%, dados que condizem com várias pesquisas realizadas por autores piauienses em áreas rurais quilombolas - Abreu (2000), Franco \& Barros (2006) - e em áreas rurais constituídas de camponeses - Aguiar (2009) e Oliveira et al. (2010), onde as espécies nativas corresponderam a uma porcentagem maior que as cultivadas em quintais e em vasos. Das espécies citadas nas três comunidades, algumas estão incluídas na lista oficial das ameaçadas de extinção pelo IBAMA (2009): aroeira (Myracrodruon urundeuva M. Allemão) e ipê-roxo (Handroanthus impetiginosus (Mart. ex DC.) Mattos), que também foram referidas por Montelles \& Pinheiro (2007).

Um total de dez espécies de plantas apresentou ampla versatilidade quanto a seus usos, com IR $>1$, sendo indicadas para até seis sistemas corporais: Curatella americana L. (1,11), Krameria tomentosa A. St.-Hil., Plechtranthus barbatus Andrews (1,17), Cymbopogon citratus (DC.) Staph $(1,44)$, Amburana cearensis (Allemão) A. C. Sm.; Lippia alba (Mill.) N. E. Br.; Mentha $x$ villosa Huds.; Ocimum gratissimum L. e Terminalia fagifolia Mart. $(1,67)$ (Tabela 1).

As espécies que apresentaram maior valor de IR (=2) foram Ximenia americana L. com 31 citações, sugerida para o combate a oito tipos de doenças em seis sistemas corporais, e Croton campestris A. St. Hil., que obteve 16 citações, sendo referida por treze informantes para curar cinco tipos de enfermidades em até cinco sistemas corporais. Uma planta com índice de importância 
relativamente alto pode sugerir uma real efetividade no tratamento da doença, pois em estudos etnobotânicos este índice facilitará a seleção de espécies para testes farmacológicos que possam vir a provar uma eficácia de seus princípios ativos (Friedman et al., 1986).

TABELA 1 - Espécies utilizadas como medicinais nas comunidades rurais Pau-Arrastado, Salinas e Resolvido em Campo Maior/PI. FP. forma de preparo: 1. maceração; 2. lambedor; 3. chá (decocto); 4. tintura; 5. suco; 6. salada; 7. garrafada; 8. in natura; 9. torrado; 10. óleo; 11. chá (infusão). FU - Forma de uso: a. inalação; b. via oral; c. escalda-pé; d. uso-tópico; e. gargarejo; f. banho. PU. parte utilizada: pi. planta inteira; fo. folha; cs. casca; ra. raiz; ca. caule; fr. fruto; fl. flor; se.semente; la. látex; bu. bulbo; IR. importância relativa.

\begin{tabular}{|c|c|c|c|c|}
\hline Família/espécie/nome vulgar & Indicação/FP & FU & $\mathbf{P U}$ & IR \\
\hline \multicolumn{5}{|l|}{ Acanthaceae } \\
\hline Justicia pectoralis var. stenophylla Leonard (trevo) & cansaço, calmante (11) & $\mathrm{b}$ & fo & 0,67 \\
\hline \multicolumn{5}{|l|}{ Anacardiaceae } \\
\hline Anacardium microcarpum Ducke. (cajuí) & $\begin{array}{l}\text { inflamação no útero }(4,7) \text {; } \\
\text { dente inflamado, } \\
\text { hemorragia bucal }(4) \text {; } \\
\text { gripe }(2,7)\end{array}$ & $\mathrm{b}$ & cs & 0,83 \\
\hline Myracrodruon urundeuva M.Allemão (aroeira) & inflamação no útero (4) & $\mathrm{b}$ & cs & 0,83 \\
\hline \multicolumn{5}{|l|}{ Amaranthaceae } \\
\hline Chenopodium ambrosioides L. (mastruz) & $\begin{array}{l}\text { gripe, inflamação (5); } \\
\text { pancada (1) }\end{array}$ & $b, d$ & fo & 0,83 \\
\hline \multicolumn{5}{|l|}{ Apocynaceae } \\
\hline Catharanthus roseus (L.) G. Don (boa-noite; bom-dia) & dor nas pernas $(4)$ & $\mathrm{d}$ & $\mathrm{fl}$ & 0,83 \\
\hline $\begin{array}{l}\text { Himatanthus sucuuba (Spruce ex Müll. Arg.) Woodson } \\
\text { (janaguba) }\end{array}$ & câncer, reumatismo (7) & $\mathrm{b}$ & la & 0,61 \\
\hline \multicolumn{5}{|l|}{ Asparagaceae } \\
\hline Asparagus sp (milindro) & nervosismo (11) & $\mathrm{b}$ & fo & 0,36 \\
\hline \multicolumn{5}{|l|}{ Asteraceae } \\
\hline Tanacetum vulgare L. (mulatinho) & dor de barriga $(3,11)$ & $\mathrm{b}$ & fo & 0,83 \\
\hline \multicolumn{5}{|l|}{ Bignoniaceae } \\
\hline Heliotropium indicum L. (crista-de-galo) & febre, gripe (3) & $b, f$ & in & 0,83 \\
\hline \multicolumn{5}{|l|}{ Capparaceae } \\
\hline Tarenaya spinosa (Jacq.) Raf. (mussambé) & gripe (2) & $\mathrm{b}$ & ra & 0,36 \\
\hline \multicolumn{5}{|l|}{ Caryocaraceae } \\
\hline Caryocar coriaceum Wittm. (pequi) & gripe (10) & $\mathrm{b}$ & fr & 0,69 \\
\hline
\end{tabular}

(continua) 
TABELA 1 - Continuação

\begin{tabular}{|c|c|c|c|c|}
\hline Família/espécie/nome vulgar & Indicação/FP & FU & $\mathbf{P U}$ & IR \\
\hline \multicolumn{5}{|l|}{ Cecropiacae } \\
\hline Cecropia glaziovi Snethl (embaúba) & rins $(4)$ & $\mathrm{b}$ & cs & 0,83 \\
\hline \multicolumn{5}{|l|}{ Cochlospermaceae } \\
\hline $\begin{array}{l}\text { Cochlospermum vitifolium (Willd.) Spreng. (algodão- } \\
\text { bravo) }\end{array}$ & dor de urina (4) & $\mathrm{b}$ & cs & 0,36 \\
\hline \multicolumn{5}{|l|}{ Combretaceae } \\
\hline Combretum duarteanum Cambess. (caatinga-branca) & bronquite (4) & $\mathrm{b}$ & cs & 0,36 \\
\hline Combretum leprosum Mart. (mufumbo) & estancar sangue (4) & d & cs & 0,83 \\
\hline Terminalia fagifolia Mart. (chapada) & intestino, fígado, dor de estômago (4) & $\mathrm{b}$ & cs & 1,67 \\
\hline \multicolumn{5}{|l|}{ Convolvulaceae } \\
\hline Ipomoea sp (salsa-branca) & doença venérea (7) & $\mathrm{b}$ & $\mathrm{ra}$ & 0,36 \\
\hline \multicolumn{5}{|l|}{ Crassulaceae } \\
\hline Bryophyllum pinnatum (Lam.) Oken (folha-santa) & cicatrização (1) & d & fo & 0,47 \\
\hline \multicolumn{5}{|l|}{ Cucurbitaceae } \\
\hline Momordica charantia L. (melão-de-são-caetano) & gastrite (3) & $\mathrm{b}$ & fo, fr & 0,61 \\
\hline Wilbrandia verticillata Cong. (batata-de-tiú) & gripe, pneumonia (4) & $\mathrm{b}$ & ra & 0,36 \\
\hline \multicolumn{5}{|l|}{ Dilleniaceae } \\
\hline Curatella americana L. (sambaíba) & $\begin{array}{l}\text { osteosporose, diabete, fígado, apendicite, dor de } \\
\text { estômago (4) }\end{array}$ & $\mathrm{b}$ & cs & 1,11 \\
\hline \multicolumn{5}{|l|}{ Euphorbiaceae } \\
\hline $\begin{array}{l}\text { Cnidoscolus loefgrenii Pax \& K. Hoffm. (cançanção- } \\
\text { branco) }\end{array}$ & coceira (7) & $\mathrm{b}$ & $\mathrm{ra}$ & 0,36 \\
\hline Croton blanchetianus Baill. (marmeleiro) & figado, intestino, rins (4) & $\mathrm{b}$ & cs & 0,72 \\
\hline Croton campestris A. St. Hil. (velame) & $\begin{array}{l}\text { intestino }(3,11) \text {; inflamação na garganta, fígado } \\
\text { (3); nascimento de dente, reumatismo (11) }\end{array}$ & $\mathrm{b}$ & ra, fo & 2,00 \\
\hline Jatropha ribifolia (Pohl) Baill. (pinhão-branco) & inflamação no útero (7) & $\mathrm{b}$ & la & 0,72 \\
\hline Ricinus communis L. (mamona) & dor de barriga, gripe (10) & $\mathrm{b}$ & fr & 0,72 \\
\hline \multicolumn{5}{|l|}{ Iridaceae } \\
\hline Cipura paludosa Aubl. (cebola-de-trovão) & dor de barriga ( 3 ) & $\mathrm{b}$ & bu & 0,36 \\
\hline \multicolumn{5}{|l|}{ Krameriaceae } \\
\hline Krameria tomentosa A.St.-Hil. (carrapicho-de-boi) & $\begin{array}{l}\text { inflamação nos rins (4); câncer (4,7); próstata, } \\
\text { pressão (4) }\end{array}$ & $\mathrm{b}$ & $\mathrm{ra}$ & 1,17 \\
\hline \multicolumn{5}{|l|}{ Lamiaceae } \\
\hline Hyptis suaveolens (L.) Poit. (bamburral) & gripe (11); sinusite (9) & $\mathrm{b}, \mathrm{a}$ & fo & 0,72 \\
\hline Leonotis nepetifolia (L.) R. Br. (cordão-de-são-francisco) & reumatismo (3) & $\mathrm{b}$ & fo & 0,36 \\
\hline Lippia gracillis Humb., Bonpl. \& Kunth.(alecrim) & gripe $(3,4)$; problema respiratório $(9)$ & $\mathrm{b}, \mathrm{a}$ & fo & 0,83 \\
\hline
\end{tabular}

(continua) 
TABELA 1 - Continuação

\begin{tabular}{l}
\hline Família/espécie/nome vulgar \\
\hline Mentha arvensis L. (vick) \\
Mentha $x$ villosa Huds (hortelã) \\
Ocimum gratissimum L. (alfavaca/manjericão) \\
Plechtranthus amboinicus (Lour.) Spreng. (malva-do- \\
reino) \\
Plechtranthus barbatus Andrews (boldo)
\end{tabular}

\section{Lecythidaceae}

Lecythis pisonis Cambess. (sapucaia)

Indicação/FP
gripe $(11,2) ;$ inflamação na garganta (11)
dor de barriga, gripe $(3,11)$; febre, inflamação na
garganta (3)

gripe (11); dor de cabeça, intestino (3)

gripe, tosse $(2,3)$

$\begin{array}{lll}\mathrm{b}, \mathrm{f} & \text { fo } & 1,67 \\ \mathrm{~b} & \text { fo } & 0,36\end{array}$

intestino (3,11); dor de cabeça (3); fígado (11) $\quad \mathrm{b} \quad$ fo $\quad 1,17$

colesterol (4)

b fo $\quad 0,36$

\section{Fabaceae}

Aeschynomene sp (canela-de-véi)

problemas respiratórios (9)

FU PU IR

Amburana cearensis (Allemão) A.C.Sm. (imburana)

Anadenanthera colubrina (Vell.) Brenan (angico-preto)

congestão nasal (9);

gripe $(2,3)$

Bauhinia flexuosa Moric (cipó-escada)

infecção (4); gripe (2)

Bauhinia ungulata L. (mororó)

hérnia de disco (4)

diabete (4)

Copaifera luetzelburgii Harms (podói)

circulação (1); pedra nos rins, próstata, inflamação na garganta(4)

Hymenaea courbaril L. (jatobá-da-mata) gripe $(2,4)$

Hymenaea stignocarpa Mart. ex Hayne (jatobá-de-porco)

Libidibia ferrea (Mart.) L. P. Queiroz (jucá)

inflamação no útero, próstata (4)

Neptunia plena Lindl. (caroba-rasteira)

gripe (2)

inflamação na garganta (7)

Plathymenia reticulata Benth (candeia)

rins (4)

Senna alata (L). Roxb. (maria-mole)

gripe (3)

Senna occidentalis (L.) Link (fedegoso)

impingem (1)

Stryphnodendron barbatiman Mart. (barbatimão)

inflamação, próstata (4)

Vachellia farnesiana (L.) Wight \& Arn (coronha)

derrame (3); intestino $(3,4)$

a, b cs, se $\quad 1,67$

b cs $\quad 0,47$

b ca $\quad 0,36$

b cs $\quad 0,83$

b ra, cs 0,83

\section{Malvaceae}

Helicteres heptandra L. B. Sm. (sacatrapo)

Luehea speciosa Willd.

(açoita-cavalo)

infecção nos rins (4)

anemia, fígado, gastrite inflamação na garganta,

hemorroida (4)

pneumonia $(7,3)$;

dor de estômago (4)

b ra

0,83

Mouriri surinamensis Aubl. (crioli)

congestão nasal (9); gripe $(2,3)$

a se, cs

0,83

afta, cicatrização, circulação, gripe, inflamação $\quad$ b $\quad$ cs $\quad 2,00$

no útero e nos rins, intestino (4) 
TABELA 1 - Conclusão

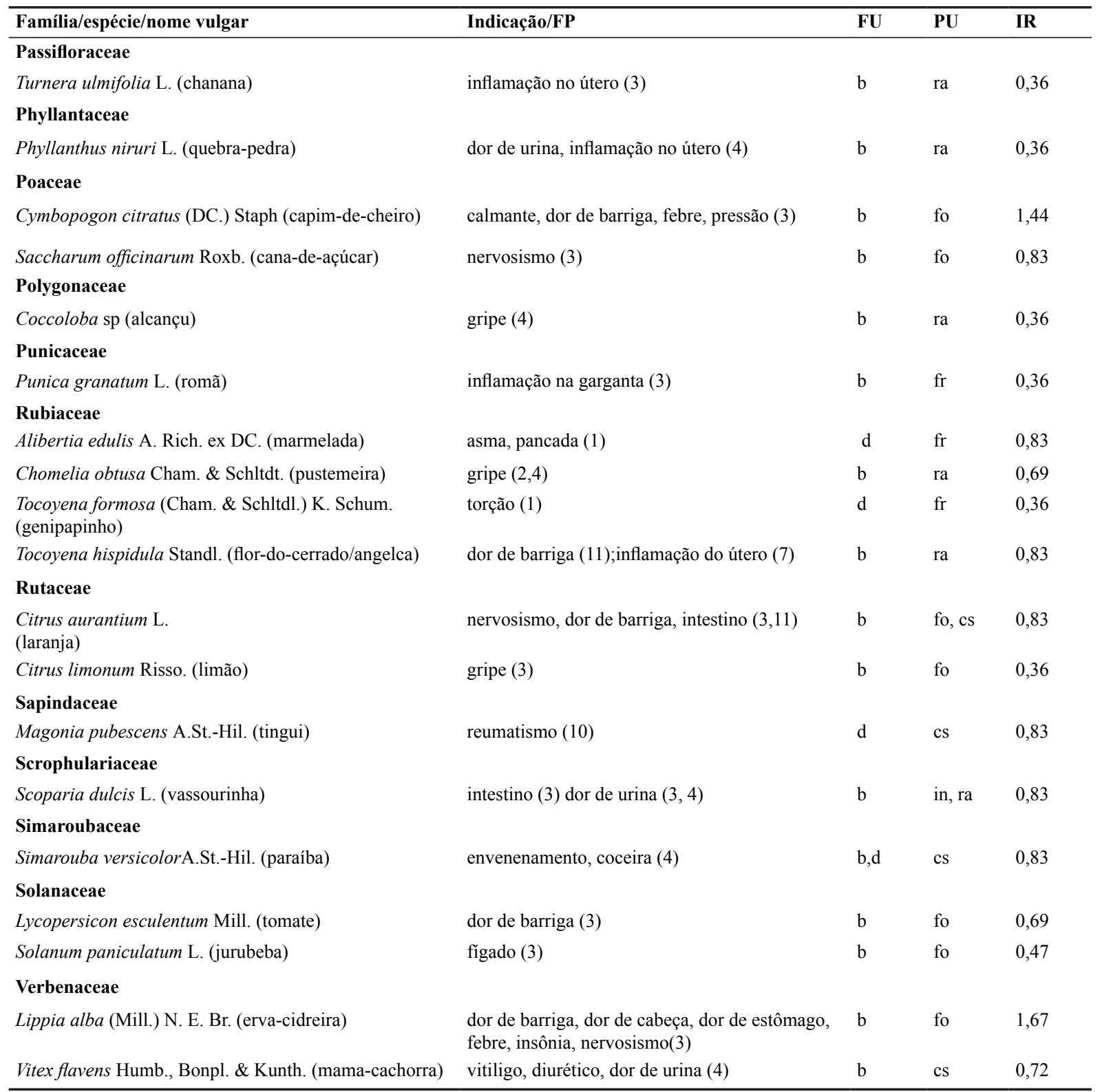

FONTE: Elaboração própria. 
A forma de preparo mais adotada foi a tintura $(38,2 \%)$, seguida de chá $(40,8 \%$, sendo por decocto $28 \%$ e infusão $12,8 \%)$, lambedor $(8,5 \%)$, garrafada $(4,5 \%)$, maceração $(2,6 \%)$, óleo $(2,1 \%)$, pó $(1,8 \%)$ e suco $(1,5 \%)$ (Figura 2). Coelho-Ferreira (2000) define tintura como um procedimento que consiste em um remédio obtido de material vegetal triturado e colocado em álcool por um período que pode variar de 7 a 15 dias. Constatou-se que as formas de preparo mais adotadas estão relacionadas com a agilidade na confecção do produto a ser consumido.

Quanto às partes das plantas empregadas para fins medicinais, as folhas foram mais utilizadas (32\%), seguidas das cascas $(31 \%)$, raízes $(16 \%)$, frutos $(9,9 \%)$, látex, plantas inteiras e sementes $(2,4 \%$ cada), bulbos, caules e flores (1,3\% cada). Castellucci et al. (2000), ao desenvolverem pesquisa com plantas medicinais em uma abordagem etnobotânica, obtiveram resultado semelhante e explicam a provável confirmação ao fato de as folhas serem acessíveis e estarem disponíveis durante a maior parte do ano.

As indicações terapêuticas das plantas medicinais nas comunidades Pau-Arrastado, Salinas e Resolvido

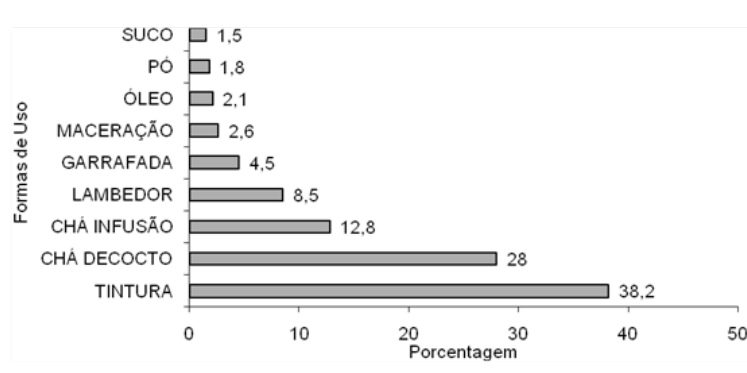

FIGURA 2 - Formas de uso das espécies para fins medicinais nas comunidades rurais Pau-Arrastado, Salinas e Resolvido, Campo Maior-PI.

FONTE: Pesquisa de campo.

foram enquadradas nos sistemas corporais constantes na Figura 3, com base na OMS (2000). O maior percentual de espécies citadas recaiu nas enfermidades relacionadas às infecções bacterianas e virais, como bronquite, gripe, inflamação na garganta e pneumonia.

A maioria das espécies levantadas no presente estudo ainda não foi estudada quanto à sua atividade biológica, demonstrando-se a necessidade de mais pesquisas com plantas em área de transição. No entanto, Carvalho

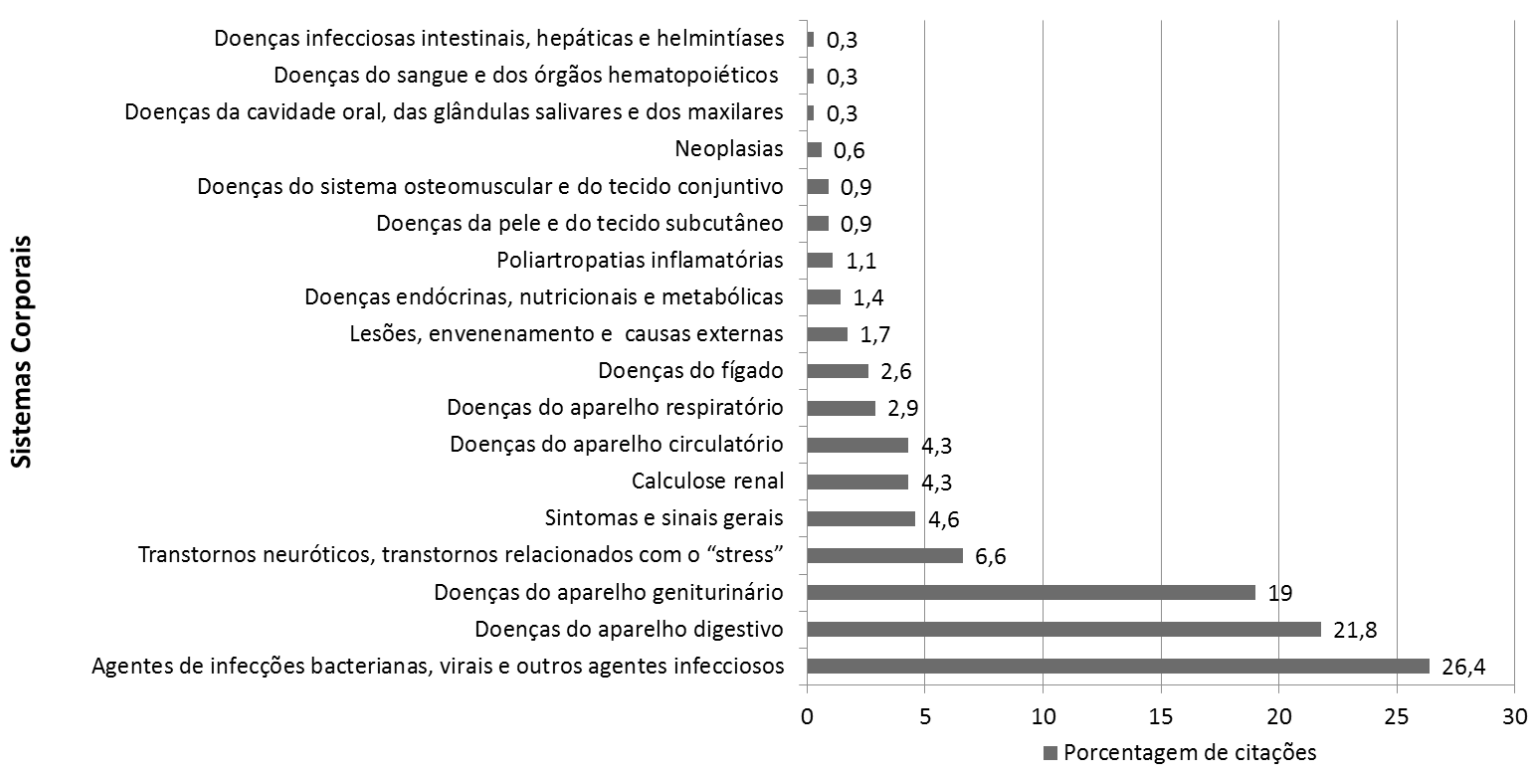

FIGURA 3 - Porcentagem de citações por Sistemas Corporais de acordo com a OMS (2000) nas Comunidades Pau-Arrastado, Salinas e Resolvido, Campo Maior/PI.

FONTE: Pesquisa de campo. 
et al. (1996) validaram cientificamente as propriedades analgésica e anti-inflamatória do jucá (Libidibia ferrea (Mart.) L.P. Queiroz) e Bacchi \& Sertie (1994) deram validação à atuação antiulcerogênica, porém na área de estudo só foi reconhecida pelos moradores a sua capacidade analgésica e anti-inflamatória. Estudos fitoquímicos e farmacológicos realizados por Stefanini et al. (2002) com Lippia alba (Mill.) N. E. Br.(erva-cidreira) comprovaram que a planta contém um princípio ativo relacionado com as ações analgésica, calmante e sedativa. É uma espécie citada pelos moradores locais de grande utilidade como calmante, contra dor de cabeça e febre, demostrando que o uso citado pelos entrevistados condiz com dados da literatura.

Salienta-se que algumas espécies medicinais citadas nas comunidades do presente estudo também foram referidas por Moreira et al. (2002) em Ilhéus (BA). Dentre as mesmas, podemos citar: Cecropia glaziovi Snethl., Curcubita pepo L., Chenopodium ambrosioides L., Cymbopogon citratus (DC.) Staph., Heliotropium indicum L., Lippia alba (Mill.) N. E. Br., Momordica charantia L., Saccharum officinarum Roxb., Scoparia dulcis L., Senna occidentalis (L.) Link. e Zea mays L., demonstrando, como citado anteriormente, que espécies exóticas que são facilmente cultivadas constam nas listagens dos artigos sobre plantas medicinais com muita frequência. Quanto às nativas, as mesmas citações de uso provavelmente demonstram a viabilidade de desenvolvimento de estudos mais aprofundados quanto à fitoquímica e à farmacognosia.

De acordo com resultados das entrevistas sobre o conhecimento passado de geração a geração, quanto ao uso dos vegetais, para $49,1 \%$ as informações foram adquiridas dos pais, $36,8 \%$ por meio da convivência com idosos da comunidade, $10,5 \%$ dos avós e para 3,5\% simultaneamente com os pais e avós. A transmissão sobre o uso tradicional dos recursos vegetais, realizada de pai para filho, é observada em diferentes culturas, como citado por Brito \& Brito (1999), Lev (2000) e Silva et al. (2014) e garantida por meio da oralidade (Morais, 2011) e das vivências concretizadas entre gerações e entre sujeitos. Diferentemente dos resultados de Oliveira et al. (2010), nenhum informante relatou ter adquirido o conhecimento sobre o uso dos vegetais a partir dos meios de comunicação. Esse evento valida o respeito ainda referido às pessoas mais velhas e consolida também a manutenção do conhecimento popular tradicional entre gerações.

Nas áreas rurais, a comunicação oral é o principal meio de transmissão do conhecimento e, para que essa ocorra, é necessário o contato intenso e prolongado dos membros mais velhos com os mais novos. Isto acontece normalmente em sociedades nas quais o aprendizado é feito pela socialização, no interior do próprio grupo doméstico e de parentesco, sem necessidade de instituições mediadoras. Percebe-se, com os resultados adquiridos, que os pais residentes nas comunidades pesquisadas - Pau-Arrastado, Salinas e Resolvido - têm garantido o conhecimento para as gerações futuras. O grau de estudo não é fundamental no conhecimento e no uso das plantas medicinais, pois foi constatado que os maiores conhecedores dentro das comunidades não são escolarizados. Não obstante, na nossa contemporaneidade muitas informações e práticas com plantas medicinais estão sendo reprimidas ao longo dos tempos, devido ao pouco diálogo entre idosos, jovens e adultos, gerando um maior consumo dos alopáticos. A influência da cultura moderna e a devastação dos ambientes naturais têm causado o deslocamento das famílias para os grandes centros urbanos e a perca das heranças culturais tem acontecido de forma gradativa.

De acordo com o consenso dos informantes das comunidades estudadas quanto à potencialidade das espécies de plantas citadas (Tabela 2), a comunidade Resolvido apresentou 12 sistemas corporais e, dentre esses, houve maior concordância nas doenças do fígado $(\mathrm{FCI}=1)$. Na comunidade Pau-Arrastado foram listados 18 sistemas corporais, sendo que o maior valor foi para transtorno neurótico $(\mathrm{FCI}=0,88)$. A comunidade Salinas reuniu 10 sistemas corporais e, dentre esses, as doenças do aparelho genitourinário apresentaram o maior valor $(\mathrm{FCI}=0,37)$, revelando que o conhecimento na comunidade começa com um processo de dispersão, episódio explicado pelas diferentes ocupações em atividades profissionalizantes dos membros da comunidade. Apesar do maior distanciamento do centro da cidade de Campo Maior, a população local mantém contato direto com outra cidade mais próxima denominada de Coivaras, e dela absorve hábitos culturais religiosos, alimentícios e de afazeres domésticos. 
TABELA 2 - Fator de Consenso dos Informantes (FCI) das plantas utilizadas como medicinais nas comunidades rurais Pau-Arrastado (PA), Salinas (S) e Resolvido (R), município de Campo Maior/PI. Legenda: $\mathrm{N}^{\circ}$ sp = número de espécies; $\mathrm{N}^{\circ}$ cit = número de citações.

\begin{tabular}{|c|c|c|c|c|c|c|c|c|c|}
\hline Categorias & $\begin{array}{l}\text { FCI } \\
(\mathrm{R})\end{array}$ & $\begin{array}{l}\mathrm{N}^{\circ} \mathrm{sp} \\
(\mathrm{R})\end{array}$ & $\begin{array}{l}\mathrm{N}^{0} \text { cit. } \\
(\mathrm{R})\end{array}$ & $\begin{array}{l}\text { FCI } \\
(\mathrm{S})\end{array}$ & $\begin{array}{l}\mathrm{N}^{\circ} \mathrm{sp} \\
(\mathrm{S})\end{array}$ & $\begin{array}{l}\mathrm{N}^{\mathrm{o}} \text { cit. } \\
(\mathrm{S})\end{array}$ & $\begin{array}{l}\text { FCI } \\
(\mathrm{PA})\end{array}$ & $\begin{array}{l}\mathrm{N}^{\circ} \mathrm{sp} \\
(\mathrm{PA})\end{array}$ & $\begin{array}{l}\mathrm{N}^{\circ} \text { cit. } \\
\text { (PA) }\end{array}$ \\
\hline Agentes de infecções bacterianas, virais & 0,25 & 13 & 17 & 0 & 8 & 8 & 0,47 & 23 & 43 \\
\hline Doenças do aparelho circulatório & 0 & 1 & 1 & 0 & 3 & 3 & 0,33 & 5 & 7 \\
\hline Doenças do aparelho respiratório & 0 & 3 & 3 & - & - & - & 0,4 & 4 & 6 \\
\hline Doenças endócrinas, nutricionais e metabólicas & 0 & 1 & 1 & - & - & - & 0 & 2 & 2 \\
\hline $\begin{array}{l}\text { Transtornos neuróticos, transtornos relacionados com o } \\
\text { "stress" }\end{array}$ & 0,42 & 5 & 8 & 0 & 2 & 2 & 0,88 & 2 & 10 \\
\hline Doenças do fígado & 1 & 1 & 2 & 0 & 1 & 1 & 0,25 & 4 & 5 \\
\hline Calculose renal & 0 & 1 & 1 & - & - & - & - & - & - \\
\hline Sintomas e sinais gerais & 0 & 5 & 5 & 0 & 2 & 2 & 0,28 & 6 & 8 \\
\hline Doenças da pele e do tecido subcutâneo & - & - & - & 0 & 1 & 1 & 0 & 1 & 1 \\
\hline $\begin{array}{l}\text { Doenças do sistema osteomuscular e do tecido } \\
\text { conjuntivo }\end{array}$ & - & - & - & 0 & 1 & 1 & 0 & 2 & 2 \\
\hline Doenças infecciosas intestinais, hepáticas e helmintíases & - & - & - & - & - & - & 0 & 1 & 1 \\
\hline Neoplasias e doenças virais & - & - & - & - & - & - & 0 & 1 & 1 \\
\hline Transtorno do sistema cardiovascular & - & - & - & - & - & - & 0 & 1 & 2 \\
\hline Transtorno do sistema nervoso & - & - & - & - & - & - & 0 & 1 & 1 \\
\hline
\end{tabular}

FONTE: Pesquisa de campo.

Houve maior consenso entre os informantes da comunidade Resolvido em comparação com as outras duas comunidades, pois, em três sistemas para as plantas indicadas no tratamento de doenças do fígado, do aparelho digestivo e do aparelho geniturinário, o valor do FCI aproxima-se de 1, indicando que essas categorias são culturalmente importantes para as comunidades estudadas. Esse dado justifica a postura mais ruralizada dos membros da comunidade, garantida pela localização geográfica, distância do centro da cidade de Campo Maior e maior envolvimento com as atividades profissionais desenvolvidas dentro da própria comunidade.

Essa análise indica que a maioria dos informantes compartilha dos mesmos conhecimentos a respeito das propriedades medicinais das plantas, existindo pouca variação na forma de produção e uso do remédio natural.

\section{Conclusão}

As comunidades rurais Pau-Arrastado, Salinas e Resolvido utilizam uma grande diversidade de plantas medicinais para a cura e a prevenção de doenças corriqueiras. O conhecimento sobre plantas medicinais vem de tradição familiar na comunidade Resolvido, enquanto que nas comunidades Pau-Arrastado e Salinas é compartilhado pelos membros idosos e adultos de forma mais intensificada do que com os jovens. As mulheres apresentaram maior conhecimento sobre espécies cultivadas ou que ocorrem em áreas ruderais próximas às residências e os homens demonstraram maior domínio sobre as plantas nativas arbóreas. Constatou-se que existem alguns fatores que são fundamentais no conhecimento e no uso das plantas medicinais e dentre eles ressaltam- 
-se as convivências familiares, os afazeres diários e as atividades profissionais. É necessário que os moradores permaneçam com seu modo de vida simples, ligado ao convívio com o ambiente natural.

Os cercados e as matas são os ambientes mais explorados para coleta e o cultivo das espécies medicinais. A maioria dos moradores reside na região desde o nascimento, levando-os a uma maior integração e adaptação à realidade local, vivendo basicamente da agricultura familiar e a pecuária. O hábito de usar plantas para o combate aos problemas corriqueiros é algo que faz parte da cultura das comunidades estudadas e em muitos casos coincide com os achados científicos ou fornecem informações valiosas quanto à escolha de novas espécies nativas passíveis de estudos de cunhos fitoquímico e farmacológico.

Neste estudo, constatou-se que existe uma forte relação cultural no uso das plantas medicinais, que ao longo dos tempos foi adquirindo maior confiança a partir das comprovações por parte dos moradores na cura

\section{Referências}

Abreu, J. R. de. Diversidade de recursos vegetais do cerrado utilizados pelos quilombolas Mimbó (Amarante, Piauí, Brasil). Dissertação (Mestrado em Biologia Vegetal) - UFPE, 2000.

Agra, M. F.; Silva, K. N; Basílio, I. J. L. D.; França, P. F.; Barbosa-Filho, J. M. Survey of medicinal plants used in the region Northeast of Brazil. Revista Brasileira de Farmacognosia, 18, 472-508, 2008.

Aguiar, L. do C. G. G. de. Etnobotânica em quintais de comunidades rurais no domínio do cerrado Piauiense (Munícipio de Demerval Lobão, Piauí, Brasil). Dissertação (Mestrado em Desenvolvimento e Meio Ambiente) - UFPI, 2009.

Aguiar, L. do C. G. G. de; Barros, R. F. M. Plantas medicinais cultivadas em quintais de comunidades rurais no domínio do cerrado piauiense (município de Demerval Lobão, Piauí, Brasil). Revista Brasileira de Plantas Medicinais, 14, 419434, 2012.

Albuquerque, C. A.; Albuquerque, U. P. Perceptions towards biological conservation in the community of Vila Velha, Pernambuco, Brazil. Interciencia, 30(8), 460-465, 2005.

Almeida, C. F. C. B. R.; Amorim, E. L. C.; Albuquerque, U. P.; Maia, M. B. S. Medicinal plants popularly used in the Xingó de doenças. Nestes dois biomas existentes no Piauí, o uso, os preparos e as credibilidades das plantas medicinais em áreas rurais são aspectos comuns. No entanto, verificou-se que a relação e o proceder do mantenedor de áreas rurais de Cerrado divergem do mantenedor do bioma Caatinga, pois nesse as partes usadas das plantas geralmente são desidratadas em decorrência dos períodos de estiagem.

\section{Agradecimentos}

Aos moradores das comunidades estudadas, pela disponibilidade durante as coletas botânicas e entrevistas e às Secretarias de Educação Estadual e Municipal de Campo Maior, pela concessão do afastamento para a realização das atividades de pesquisa. Aos professores Dr. Carlos Hiroo e Dra. Socorro Lira, pelas imensas contribuições ao trabalho.

region - a semi-arido location in Northeastern Brazil. Journal of Ethnobiology and Ethnomedicine, 2(15), 1-9, 2006.

Amorozo, M. C. de. M. A abordagem etnobotânica na pesquisa de plantas medicinais. In: Di Stasi, L. C. (Org.). Plantas medicinais: arte e ciência - um guia de estudo interdisciplinar. São Paulo: Editora UNESP, p. 47-68, 1996.

APG III. Angiosperm Phylogeny Group III. An update of the phylogeny group classification for the orders and families of flowering plants. Botanical Journal of the Linnean Society, 161(2), 105-121, 2009.

Appolinário, F. Introdução à análise quantitativa de dados. In: Appolinário, F. (Org.). Metodologia científica: filosofia e prática da pesquisa. São Paulo: Thomson Leaming, 2006.

Bacchi, E. M.; Sertie, J. A. A. Antiulcer action of Styrax camporum and Caesalpinia ferrea in rats. Planta Medica, 60(2), 118-120, 1994.

Barros, J. S.; Castro, A. A. J. F.; Farias, R. R. S. de. Vegetação e solos no Complexo de Campo Maior-PI: níveis de interações. In: Lopes, W. G. R.; Gomes, J. M. A.; Moita Neto, J. M.; Araújo, J. L. L.; Leite, L. F. C.; Nascimento, P. W. G.; Barros, R. 
F. M. (Orgs). Cerrado piauiense: uma visão multidisciplinar. Teresina: EDUFPI, p. 195-222, 2007.

Begossi, A.; Leitão Filho, H. F.; Richerson, P. J. Plant uses in a Brazilian coastal fishing community (Buzios Island). Journal of Ethnobiology, 13(2), 233-256, 1993.

Bernard, H. R. Research in cultural anthropology. Newbury Park, CA, EEUU: Sage, 1988.

Bennet, B. C.; Prance, G. T. Introduced plants in the indigenous pharmacopoeia of Northen South America. Economic Botany, 54(1), 90-102, 2000.

Brasil. Ministério da Saúde. Secretaria de Ciência, Tecnologia e Insumos Estratégicos. Departamento de Assistência Farmacêutica. Política Nacional de Plantas Medicinais e Fitoterápicos. Brasília, 2006. Disponível em: <http://bvsms.saude.gov.br/ bvs/publicacoes/politica_nacional_fitoterapicos.pdf $>$ Acesso em 23 dez. 2012.

Brito, A. R. M. S.; Brito, A. A. S. Forty years of Brazilian medicinal plant research. Journal of Ethnopharmacology, 39(1), 53-67, 1993. doi: 10.1016/0378-8741(93)90050-F

Brito, A. R. M. S.; Brito, A. A. S. Medical plant research in Brasil: data from regional and national meeting. In: Balick, M. J.; Elisabetsky, E.; Laird, S. A. (Eds.). Medicinal Resources of the tropical forest: biodiversity and its importance to human health. New York: Columbia University Press, 1999, p. 386-401.

Carvalho, J. C. T.; Teixeira, J. R. M.; Souza. P. J. C., Bastos, J. K.; Filho, D. S.; Sarti, S. J. Preliminary studies of analgesic and anti-inflammatory properties of Caesalpinia ferrea crude extract. Journal of Ethnopharmacology, 53, 175-178, 1996. doi:10.1016/0378-8741(96)01441-9

Castellucci, S.; Lima, M. I. S.; Nordi Marques, J.G. W. Plantas medicinais relatadas pela comunidade residente na estação ecológica de Jataí, Município de Luís Antônio/SP: uma abordagem etnobotânica. Revista Brasileira de Plantas medicinais, 3(1), 51-60, 2000.

Coelho-Ferreira, M. R. Identificação e valorização das plantas medicinais de uma comunidade pesqueira do litoral paraense (Amazônia brasileira). Belém, Tese (Doutorado em Ciências Biológicas) - UFPA, 2000.

Di Stasi, L. C. (Org.). Plantas medicinais: arte e ciência - um guia de estudo interdisciplinar. São Paulo: Editora UNESP, 1996.

Franco, E. A. P. A.; Barros, R. F. M. Uso e diversidade de plantas medicinais no Quilombo Olho D’Água dos Pires,
Esperantina, Piauí. Revista Brasileira de Plantas Medicinais, 8(3), 78-88, 2006.

Freitas, A. V. L.; Coelho, M. de. F. B.; Maia, S. S. S.; Azevedo, A. R. B de. A. Plantas medicinais: um estudo etnobotânico nos quintais do Sítio Cruz, São Miguel, Rio Grande do Norte, Brasil. Revista Brasileira de Biociências, Porto Alegre, 10(1), 48-59, 2012.

Friedman, J.; Yaniv, Z.; Dafni, A.; Pale-Witch, D. A. A preliminary classification of the healing potential medicinal plants, based on a rational analysis of in ethnopharmacology field survey among Bedouins in the Negev desert, Israel. Journal of Ethnopharmacology, 16, 275-287, 1986. doi: 10.1016/03788741(86)90094-2

Fujita, T.; Sezik, E.; Tabata, M.; Yesilada, E.; Honda, G.; Takeda, Y.; Tanaka, T.; Takaishi, Y. Traditional medicine in Turkey VII. Folk medicine in middle and west black sea regions. Economic Botany, 49, 406-422, 1995.

Giraldi, M.; Hanazaki, N. Uso e conhecimento tradicional de plantas medicinais no Sertão do Ribeirão, Florianópolis, SC, Brasil. Acta Botanica Brasílica, 24(2), 395-406, 2010.

Guarim Neto, G.; Maciel, M. R. A. O saber local e os recursos vegetais em Juruena: Mato Grosso. Cuiabá, MT: Entrelinhas; EdUFMT, 2008.

Haller-Jr., J. S. The United States Pharmacopoeia: Its Origin and revision in the 19th century. Bulletin of the New York Academy of Medicine, 58, 480-492, 1982.

IBAMA - Instituto Brasileiro do Meio Ambiente e dos Recursos Naturais Renováveis. Ecossistemas brasileiros: ocupação da Amazônia. Disponível em: <http://www.ibama.gov.br/ecossistemas/ocupacao.htm>. Acesso em: 24 abr. 2009.

IBGE - Instituto Brasileiro de Geografia e Estatística. Aspectos físicos de Campo Maior. Disponível em: <http://www.ibama. gov.br/ecossistemas/ocupacao.htm>. Acesso em: 25 set. 2008.

IBGE - Instituto Brasileiro de Geografia e Estatística. Pesquisa mensal de empregos: indicadores. Piauí: Disponível em: <http:/www.ibama.gov.br/ecossistemas/ocupacao.htm>. Acesso em: 23 out. 2009.

Kruel, V. S. F.; Peixoto, A. L. Etnobotânica na Reserva Extrativista Marinha de Arraial do Cabo. Acta Botanica Brasilica, 18, 177-190, 2004.

Lev, E. A. Etnhopharmacological survey of traditional drugs sold in Israel at the end of $20^{\text {th }}$ century. Jornal of Ethnopharmacology, 72, 191-205, 2000. doi: 10.1016/S03788741(00)00230-0 
Maciel, M. A. M.; Pinto, A. C.; Veiga Júnior, V. E. Plantas medicinais: a necessidade de estudos multidiciplinares. Química Nova, 25(3), 429-438, 2002.

MOBOT. Disponível em: <http://www.ipni.org>. Acesso em: 10 jun. 2014. (www.mobot.org).

Montelles, R.; Pinheiro, C. U. Plantas medicinais em um quilombo maranhense: uma perspectiva etnobotânica. Revista de Biologia e Ciências da Terra, 7(2), 38-48, 2007.

Morais, V. M. Etnobotânica nos quintais da comunidade de Abderramant em Caraúbas - RN. Mossoró, Tese (Doutorado em Agronomia: Fitotecnia) - UFERSA, 2011.

Moreira, R. C. T.; Costa, L. C. D. B.; Costa, R. C. S.; Rocha, E. A. 2002. Abordagem etnobotânica acerca do uso de plantas medicinais na Vila Cachoeira, Ilhéus, Bahia, Brasil. Acta Farmacéutica Bonaerense, 21(3), 205-211, 2002.

Mori, S. A.; Silva, A. M.; Lisboa G.; Coradim, L. Manual de manejo do Herbário Fanerogâmico. 2. ed. Ilhéus: CEPLAC, 1989.

Oliveira, F. C. S.; Barros, R. F. M.; Moita Neto, J. M. Plantas medicinais utilizadas em comunidades rurais de Oeiras, semiárido piauiense. Revista Brasileira de Plantas Medicinais, Botucatu, 12(3), 282-301, 2010.

OMS - Organização Mundial da Saúde. Classificação estatística internacional de doenças e problemas relacionados à saúde. São Paulo: Editora da Universidade de São Paulo, 2000.

Pasa, M. C.; Soares, J. J.; Guarim-Neto, G. Estudo etnobotânico na comunidade de Conceição-Açu (alto da bacia do rio Aricá Açu, MT, Brasil). Acta Botanica Brasilica, 19(2), 195-207, 2005.

Paulilo, M. I. S. Movimentos das mulheres agricultoras e os muitos sentidos da "igualdade de gênero". In: Maçano, B.; Medeiros, L. S.; Paulilo, M. I. S. (Orgs.). Lutas camponesas contemporâneas: condições, dilemas e conquistas. São Paulo: Editora Nesp; Brasília: Núcleo de Estudos Agrários e Desenvolvimento Rural, p. 179-201, 2009.

Pereira, Z. V.; Gomes, C. F.; Lobtchenko, G.; Gomes, M. E. S.; Simões, P. D. A.; Saruwatari, R. P. S.; Rigo, V. F.; Cordeiro, W. P. Levantamento das plantas medicinais do cerrado sensu stricto da Fazenda Paraíso - Dourados, MS. Revista Brasileira de Biociências, 5(1), 249-251, 2007.
Rajakumar, N; Shivanna, M. B. Ethno-medicinal aplication of plants in the eastern region of Shimoga district, Karnataka, India. Jornal of Ethnopharmacology, 126, 64-73, 2009. doi: 10.1016/j.jep.2009.08.010

Rocha, J. A.; Neffa, E.; Leandro, L. A. L. A contribuição da Etnobotânica na elaboração de políticas públicas em meio ambiente - um desafio na aproximação do discurso à prática. Ambiência, 10(1), 43-64, 2014. doi: 10.5935/ambiencia.2014.01.04

Schweickardt, K. H. S. C. (Org.). Projeto de Pesquisa Mulheres da Floresta: memória, território e políticas públicas nas várzeas do Amazonas. Manaus: UFAM/CNPq, 2010.

Silva, A. J. R.; Andrade, L. H. C. Etnobotânica nordestina: estudo comparativo da relação entre comunidades e vegetação na Zona do Litoral. Acta Botanica Brasilica, 19, 45-60, 2005.

Silva, C. S. P.; Proença, C. E. B. P. Uso e disponibilidade de recursos medicinais no município de Ouro Verde de Goiás, GO, Brasil. Acta Botanica Brasilica, 22(2), 481-492, 2008.

Silva, M. P. da; Barbosa, F. S. Q.; Barros, R, F. M de. Estudo taxonômico e etnobotânico sobre a família Asteraceae (Dumortier) em uma comunidade rural no Nordeste do Brasil. Revista Gaia Scientia, 8, 110-123, 2014.

Sousa, R. S.; Hanazaki, N.; Lopes, J. B.; Barros, R. F. M. Are Gender and Age Important in Understanding the Distribution of Local Botanical Knowledge in Fishing Communities of the Parnaíba Delta Environmental Protection Area? Ethnobotany Research \& Applications, 10, 551-559, 2012.

Stefanini, M. B.; Rodrigues S. D.; Ming, L. C. Ação de fitorreguladores no crescimento da erva-cidreira-brasileira. Horticultura Brasileira, 20(1), 18-23, 2002.

Trotter, R.; Logan, M. Informant consensus: a new approach for identifying potentially effective medicinal plants. In: Etkin, N. L. (Ed.). Indigenous medicine and diet: biobehavioural approaches. New York: Redgrave Bedford Hills, 1986, p. 91-112.

Velloso, A. L.; Sampaio, E. V. S. B.; Pareyn, F. G. C. Ecorregiões propostas para o bioma Caatinga. Recife: PNE, 2002. 76 . Resultados do seminário de Planejamento Ecorregional da Caatinga/Aldeia-PE. 28 a 30 de novembro, 2001. 